\section{DIACRONIE}

\section{Diacronie}

Studi di Storia Contemporanea

$N^{\circ} 13,1 \mid 2013$

Contrabbandieri, pirati e frontiere: per una storia delle pratiche informali nell'America Centrale (XVII-XXI

secolo)

\title{
Contrabbandieri, banditi e guardiani
}

Le vie del traffico illecito nella costruzione del confine tra Messico e Stati Uniti

\section{Valentina Abalzati}

\section{(2) OpenEdition Journals}

\section{Edizione digitale}

URL: http://journals.openedition.org/diacronie/756

DOI: 10.4000/diacronie.756

ISSN: 2038-0925

Editore

Association culturelle Diacronie

Notizia bibliografica digitale

Valentina Abalzati, «Contrabbandieri, banditi e guardiani », Diacronie [Online], № 13, 1 | 2013,

documento 10, Messo online il 01 avril 2013, consultato il 19 avril 2019. URL : http://

journals.openedition.org/diacronie/756 ; DOI : 10.4000/diacronie.756 


\title{
Diacronie
}

N. 13 | 1|2013 Contrabbandieri, pirati e frontiere

\section{0/}

\section{Contrabbandieri, banditi e guardiani Le vie del traffico illecito nella costruzione del confine tra Messico e Stati Uniti}

\author{
Valentina ABALZATI*
}

L'articolo si propone di analizzare il processo di costruzione del confine tra Messico e Stati Uniti attraverso la lente del contrabbando, considerato il doppio filo di unione e divisione che ancora oggi determina le relazioni tra i due paesi dell'America del Nord. Il periodo considerato è quello tra il 1821, anno in cui il Messico conquistò l'indipendenza dalla Spagna, ed il 1924, quando, con la creazione della Border Patrol, l'infrastruttura di controllo del confine acquisì la forma che, mantiene tutt'ora, sebbene in scala. Nel mezzo secolo trascorso tra le travagliate vicende della guerra del 1846-1848 contro gli Stati Uniti ed il 1910, anno che sancì la fine del regime di Porfirio Díaz, si diede quel processo che portò alla definizione e al consolidamento di quella "linea sulla sabbia" che sancì la cessione al vicino del nord di un terzo del territorio messicano.

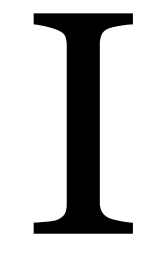

1 processo di state-building in Messico, scandito dal ritmo autoritario dell'era del Porfiriato, era riuscito a colmare un vuoto politico incorporando per la prima volta la periferia settentrionale nella giurisdizione dello Stato centrale, ponendo fine al lungo periodo di isolamento geografico della frontiera. Tuttavia, contemporaneamente alla "chiusura" del confine, si era pienamente delineato il carattere storicamente particolare della fascia fronteriza, dove un'economia regionale saldamente vincolata a quella statunitense, una rete ferroviaria che già nel 1890 connetteva tutti i dinamici centri industriali statunitensi con le zone settentrionali del 
Messico e l'emergere di una nuova classe imprenditoriale, contribuivano a farne una regione a sé stante, appendice economica per gli Stati Uniti, avanguardia capitalista per il Messico. All'impulso di separazione scaturito dalla cristallizzazione del confine corrispondeva però anche un movimento contrario. La primissima, fondamentale, forma di comportamento umano che contribuì, paradossalmente sfidandolo, a segnare il confine, fu proprio il contrabbando.

La «contorta linea che per 2000 miglia attraversa artificialmente un paesaggio arido» ${ }^{1}$ marcando il confine tra Messico e Stati Uniti fu il prodotto del convulso rapporto sviluppatosi tra i due paesi a partire dal 1821, anno dell'indipendenza della colonia spagnola. Durante il XIX secolo le interazioni tra il Messico e il suo vicino del Nord furono percorse da molteplici tensioni. Innanzitutto l'ex colonia ispanica viveva un periodo di forte instabilità interna. Il processo di costruzione del nascente Stato era scosso in primo luogo da continui tumulti e lotte intestine tra fazioni liberali e conservatrici, e in secondo luogo dalla frattura scomposta tra le forze centraliste e le pulsioni federaliste nelle regioni settentrionali del paese. Se fino alla conclusione della guerra con gli Stati Uniti nel 1848 la presenza dello Stato rimase debole, tra il 1855 e il 1880, fase culminante dei potentati dei caudillos settentrionali, essa può definirsi inesistente $^{2}$. La frammentazione politica interna rendeva inoltre particolarmente insidiosa la pressione esercitata dall'esterno: da un lato l'instancabile ricerca di influenza politica ed economica da parte delle potenze europee, infrantasi nel $1867 \mathrm{con}$ la deposizione di Massimiliano d'Asburgo e la fine del sogno di Napoleone III di un impero latino-americano da contrapporre al dominio anglosassone; dall'altro lo slancio "eccezionalista" dell'espansionismo statunitense. Gli Stati Uniti sarebbero presto passati dal sostegno indiretto alle forze politiche liberali messicane ad un'attitudine più marcatamente interventista, coerente rispetto alla tendenza espansionistica di quell'epoca che si esprimeva nell'idea "turneriana" di frontiera come «cresta dell'onda avanzante»3.

Le terre spopolate del Tejas ricevevano un gran numero di coloni angloamericani già dagli inizi dell'Ottocento. Tali spostamenti rispondevano a incentivi economici elargiti dallo stesso governo messicano (che manteneva una politica avviata dall'amministrazione coloniale), ansioso di rendere più produttive le lande desolate del settentrione. L'equilibrio demografico ed economico della zona di frontiera mutò

\footnotetext{
${ }^{1}$ TORRES-MORA, Juan, The Making of the Mexican Border. The State, Capitalism, and Society in Nuevo León, 1848-1910, Austin, University of Texas Press, 2001, p. 7.

${ }^{2}$ Ibidem, p. 8.

${ }^{3}$ Cfr. PASTORE Alessandro, Confini e frontiere nell'età moderna. Un confronto fra discipline, Milano, Franco Angeli, 2007, p. 12.
} 
lentamente a favore degli avventurosi impresari statunitensi, favoriti dalla lontananza e dall'incapacità di Città del Messico nel controllare l'area. Se nel Tejas del 1821 vivevano 3.000 persone, la popolazione del Texas del 1836, gonfiata dall'immigrazione proveniente da nord, ammontava già a più di 38.000 abitanti4. Per tentare di controllare lo squilibrio demografico, nel 1830 il Messico proibì l'immigrazione angloamericana, esacerbando gli animi indipendentisti. Questi ultimi, supportati da diversi gruppi solidali con la causa autonomista texana negli Stati Uniti, dichiararono l'indipendenza il 6 marzo 18365. Durante tutto il decennio successivo, gli sforzi centripeti del governo messicano non fecero che sobillare rivendicazioni federaliste e ribellioni rurali in tutto il nord, dove una grossa fetta di popolazione protestava contro la soppressione degli ayuntamientos (centri di potere locali) e contro l'aumento delle tasse $^{6}$. Prima ancora che gli Stati Uniti annettessero il Texas nel 1845, l'asimmetria tra i due paesi era già evidente: a sud arrancava un paese dal territorio sconfinato che poteva contare su risorse scarse, privo di un timone politico stabile ed abitato da appena sette milioni di persone; a nord si estendeva un paese dinamico, popolato da venti milioni di abitanti e ricco di risorse materiali. Il reddito complessivo prodotto dai messicani ammontava all'8\% di quello generato dai vicini settentrionali7. Gli Stati Uniti del 1845 erano un paese proteso verso la realizzazione del manifest destiny, secondo l'incisiva espressione coniata proprio quell'anno da John O'Sullivan, il quale dalle pagine della «Democratic Review» affermava

\footnotetext{
the right of [America's] manifest destiny to overspread and to possess the whole of the continent which Providence has given us for the development of the great experiment of liberty and federative self-government entrusted to us. It is a right such as that of the tree to the space of air and earth suitable for the full expansion of its principle and destiny of growth ${ }^{8}$.
}

L'arrivo alla presidenza degli Stati Uniti di un dichiarato espansionista, James K. Polk, precipitò gli eventi. Nel 1846 gli Stati Uniti dichiararono guerra al Messico e ne invasero il territorio. Nel gennaio 1847 la linea di confine aveva già compiuto un bel balzo verso sud, fagocitando senza quasi incontrare resistenza la California e il New

4 UNITED STATES GENERAL ACCOUNTABILITY OFFICE, Treaty of Guadalupe Hidalgo. Findings and Possible Options Regarding Longstanding Community Land Grant Claims in New Mexico, GAO-04-59, june 2004, p. 24

5 HERRERA, Gerardo (coord.), Nueva História Mínima de México Illustrada, México D.F., El Colegio de México, 2008, p. 284.

${ }^{6}$ Ibidem.

7 MORA-TORRES, Juan, op. cit., p. 9.

8 BRINKLEY, Alan, American History: A Survey, McGraw Hill College, 1999, p. 430. 
Mexico. D’altronde il destino del vicino del sud sembrava scontato fin dal principio: «il suo armamento era obsoleto; i suoi ufficiali, poco professionali; i suoi soldati, improvvisati»9. Il 14 settembre dello stesso anno sul Palacio Nacional di Città del Messico sventolava la bandiera statunitense e pochi mesi dopo i due paesi negoziavano il Trattato di Guadalupe Hidalgo, che dal 1848 avrebbe sancito in via definitiva l'attuale confine tra Messico e Stati Uniti. In virtù di questo trattato il Messico riconobbe la cessione di più di metà del proprio territorio (comprendente, oltre agli odierni stati della California e del New Mexico, l'Arizona e parti di Nevada e Colorado) dietro un indennizzo di quindici milioni di pesos.

La nazione messicana usciva da questa guerra profondamente segnata non solo dal punto di vista materiale, ma anche e soprattutto, da quello simbolico. Il trattato di Guadalupe Hidalgo divenne un potente catalizzatore del nazionalismo e dell'antiamericanismo messicano per i decenni a venire, monito dell'inaffidabilità e spregiudicatezza del vicino del nord ${ }^{10}$. Il lascito della guerra del 1846-1848 ebbe ripercussioni considerevoli sulle relazioni bilaterali e sugli sviluppi sociali, politici ed economici di quella larga fascia di territorio fronterizo, che nella proposta del generale Santa Anna, in fase di negoziazione del Trattato, doveva costituire una buffer zone che separasse i due Stati ${ }^{11}$.

Oltre al trauma nazionale causato dall'emorragia territoriale, lo spostamento del confine sancito dal Trattato del 1848, cristallizzato definitivamente nel Trattato di Gadsden del 1853, apriva lo spinoso problema dello status legale e delle proprietà dei centocinquantamila cittadini messicani e dei centottantamila nativi americani ritrovatisi improvvisamente in terra statunitense. Negli anni a venire si susseguirono contestazioni, interpretazioni ambigue del Trattato e violazioni vere e proprie, favorite sia dalle difficoltà culturali e linguistiche incontrate dagli ispanici in terra angloamericana, che da modi diversi e conflittuali di concepire e gestire la proprietà della terra. In uno scenario caotico come quello della California del post-1848, sconvolta dalla scoperta dell'oro e dalla gold rush che immediatamente ne scaturì, i conflitti erano semplicemente inevitabili: xenofobia, nativismo, residui di patriottismo e razzismo si tradussero in violenti scontri tra gli immigrati anglofoni e il resto della popolazione residente ${ }^{12}$. La lunga lotta per il controllo della terra tra Anglos e Mexicans

\footnotetext{
9 HERRERA, Gerardo (coord.), op. cit., p. 290.

10 DOMINGUEZ, Jorge, DE CASTRO, Rafael, The United States and Mexico. Between Partnership and Conflict, New York, Routledge, 2001, p. 9.

11 GRISWOLD DEL CASTILLO, Richard, The Treaty of Guadalupe Hidalgo: a Legacy of Conflict, Norman, University of Oklahoma Press, 1990, p. 63.

12 Ibidem, p. 67.
} 
fu costellata da numerosi episodi violenti. Tra questi basti ricordare la Cortina Rebellion nell'area di Matamoros-Brownsville negli anni Cinquanta e Sessanta del XIX secolo e la El Paso Salt War nel decennio successivo, avvenimenti di particolare rilievo intercalati in un tessuto di conflittualità persistente, fatto di una sequela interminabile di piccole battaglie, linciaggi, rivolte ${ }^{13}$. Al 1910 la gran parte dei Tejanos aveva perso parte o tutto il proprio patrimonio terriero a seguito degli sforzi di pacificazione e di costruzione dell'ordine anglo, basati su una combinazione di metodi alquanto aggressivi che comprendevano imbrogli, furti, frode e minacce ${ }^{14}$. Le contestazioni e le rivendicazioni territoriali continuarono negli anni, mai del tutto sopite, a dimostrazione del carattere storicamente "non dato" del confine tra Messico e Stati Uniti, una linea definita in modo lento, progressivo e conflittuale, emersa da lustri di accumulati atti costituitivi. Per decenni esso rimase, con le parole di Dunn, «una costruzione sociale fragile, stabilita e mantenuta con la forza»15. Le borderlands dei decenni successivi alla guerra messicano-statunitense erano terre di violenza persistente: banditismo, scorrerie indiane e ripetuti tentativi insurrezionali di stabilire repubbliche indipendenti caratterizzavano quella che venne poi definita «the Golden Age of filibustering» ${ }^{16}$.

Fino a quando, nel corso del XX secolo, il confine non divenne quella fortificata linea politica, economica e sociale che oggi conosciamo, la gran parte della popolazione della regione fronteriza spesso ignorava o contestava attivamente quella linea, che veniva percepita come imposta dalla popolazione anglofona e dallo Stato. Fu solo nel 1910, al termine di una lunga fase di restaurazione statale promossa da Porfirio Díaz, che la fluida frontier poteva dirsi ormai cristallizzata nel più solido border e che alla precisa definizione del confine politico corrispose un certo controllo del territorio. Il processo di state-building in Messico, scandito dal ritmo autoritario dell'era del Porfiriato $^{17}$, era riuscito a colmare un vuoto politico incorporando per la prima volta la periferia settentrionale nella giurisdizione dello Stato centrale, ponendo fine al lungo periodo di isolamento geografico della frontiera. Tuttavia, contemporaneamente alla cristallizzazione del confine, si era pienamente delineato il carattere particolare della

\footnotetext{
${ }_{13}$ Ibidem, p. 83

14 Ibidem.

15 DUNN, Timothy, The militarization of the U.S.-Mexico Border 1978-1992, Austin, University of Texas, CMAS Books, 1996, p. 6.

${ }^{16}$ MARTINEZ, Oscar J., Filibustering and Racism in the Borderlands, in MARTINEZ, Oscar J. (ed.), U.S.-Mexico Borderlands. Historical and Comparative Perspectives, Wilmington, Jaguar Books on Latin America, 1996, pp. 46-49.

17 Durante il lungo periodo di governo autoritario ma stabile di Porfirio Díaz (1876-1910) il Messico seppe attrarre ingenti investimenti stranieri, che contribuirono a creare la rete ferroviaria e a porre le fondamenta dell'industrializzazione del paese. Il processo di meccanizzazione dell'agricoltura e di privatizzazione delle terre tuttavia favorì la concentrazione della proprietà e il riorientamento della produzione agricola all'esportazione.
} 
fascia fronteriza. Un'economia regionale saldamente vincolata a quella statunitense, una rete ferroviaria che già nel 1890 connetteva tutti i dinamici centri industriali statunitensi con le zone settentrionali del Messico e l'emergere di una nuova classe imprenditoriale, contribuivano a farne una regione a sé stante, una "terza nazione" così diversa e incomprensibile se osservata da Washington o da Città del Messico, appendice economica per il nord, avanguardia capitalista per il sud ${ }^{18}$.

L’impulso di separazione che scaturiva dalla progressiva definizione del confine e dall'inesorabile affermarsi di due sovranità nazionali sempre più solide era però sospinto da un movimento contrario. La primissima, fondamentale, forma di comportamento umano che contribuì, paradossalmente sfidandola, a segnare quella "linea sulla sabbia” era stato proprio il contrabbando. Si può affermare che il processo di integrazione economica tra il Messico (o meglio detto, la zona settentrionale del Messico) e gli Stati Uniti, avviato con l'indipendenza dalla corona spagnola del $\mathbf{1 8 2 1}$, procedette per tutto il secolo in gran parte su binari clandestini. In quella fase storica l'intraprendenza individuale riempiva gli spazi trascurati dalle istituzioni nazionali: «it was borderlanders, rather than states, who shaped social relations within the Mexican borderlands ${ }^{19}$. Le burrascose relazioni politiche tra il giovane Stato messicano e gli Stati Uniti non avevano ostacolato la fioritura, attraverso la frontiera, di fruttuosi rapporti economici. Se dal 1848 tra Messico e Stati Uniti esisteva formalmente un confine a definire le rispettive sfere di sovranità, così come gli spazi di cittadinanza, per almeno mezzo secolo la mobilità attraverso quella linea di separazione fu pressoché illimitata. La regione di frontiera, economicamente periferica rispetto al fulcro produttivo statunitense e flebilmente legata al centro politico messicano, «a place of open spaces and expressions of freedom» ${ }^{20}$, era una zona marginale troppo lontana e non abbastanza rilevante per essere efficacemente amministrata. Considerata la mancanza generalizzata di controlli, e poiché «one side of the river always had something that was lacking on the other side» ${ }^{21}$, il confine offriva opportunità abbondanti a chiunque, su entrambe le sponde del Río Grande, fosse dotato di minime abilità imprenditoriali. Fu così che per tutta la seconda metà dell'Ottocento fino allo scoppio della Rivoluzione Messicana la spina dorsale del commercio transfrontaliero fu costituita da traffici illegali.

\footnotetext{
18 MORA-TORRES, Juan, op. cit.

19 MORA-TORRES, Juan, op. cit., p. 8

20 PAYAN, Tony, The three U.S.-Mexico border wars: drugs, immigration, and Homeland Security, Westwood, Greenwood Publishing, 2006, p. 6.

${ }^{21}$ Ibidem, p. 8.
} 
Il contrabbando, inteso sia come commercio di un bene illegale che come commercio illegale di un bene di per sé lecito, si produce in quella nicchia interstiziale che giace tra l'autorità - propria dello Stato - di istituire la legge e la sua capacità di farla rispettare: uno spazio dove ciò che è proibito ricade nell'ambito definitorio e criminalizzante dello Stato, ma non soggiace all'applicazione della legge da parte di quest'ultimo $^{22}$. Lo spazio di «nonenforcement of the law» 23 è il campo in cui il trafficante si contende con lo Stato lo spazio di manovra: il primo preme per conquistare più territorio ed il secondo lotta per la difesa del margine di legalità. Nelle borderlands messicano-americane dell'Ottocento (fino agli inizi del Novecento), terra di nessuno e lontana da tutti, lo spazio tra il paletto legale piantato dallo Stato a sancire l'illegalità di determinati beni e la capacità "poliziesca" di sorvegliare e far rispettare il divieto era enorme. Sulla geometria, sui confini di questo spazio in cui il contrabbando si muove, incide vivamente l'azione dello Stato, che tracciando la linea tra ciò che è lecito produrre e commerciare e ciò che invece non lo è, ne inibisce o fomenta il traffico. Il sistema statale di divieti, tariffe e quote non agisce direttamente sulla struttura di domanda e di offerta di una merce, piuttosto la colloca fuori (o dentro) la legge e ne altera la redditività commerciale. Così sia la natura dei beni contrabbandati, che la dimensione e l'orientamento dei flussi si trasformano storicamente, in virtù dei mutamenti dei desideri umani che mettono in moto le attività economiche e dell'evoluzione dei sistemi legali nel tempo. In una sorta di capovolgimento di prospettiva, spesso accade che il contrabbandiere non sia altro che un agente economico operante in un «business that happens to be illegal»24, a causa della posizione della linea che distingue il lecito dall'illecito, piazzata dalla legge di uno Stato che, in una data congiuntura, «makes criminals of otherwise law-abiding citizens» 25 . Le pratiche illegali dunque, più che appannaggio di una categoria di agenti socialmente devianti e intrinsecamente outlaw, appaiono come un'opzione a disposizione di vari gruppi di persone in determinati momenti, che a seconda delle opportunità scelgono $o$ meno di intraprendere.

Per gran parte del XIX secolo la struttura delle opportunità lungo la frontiera messicano-statunitense spingeva il flusso illecito dei beni, al contrario di ciò che avviene ai giorni nostri, per lo più da nord a sud. Caffè, stoffe e qualsiasi tipo di

22 ANDREAS, Peter, Border Games: policing the U.S.-Mexico divide, New York, Cornell University Press, 2000, p. 22.

23 Ibidem, p. 23.

24 Ibidem, p. 21.

25 «Failed states and failed policies. How to stop the drug wars», in The Economist, 5 march 2009. 
prodotto per cui esistesse uno sbocco di mercato era contrabbandato in Messico, evitando le restrizioni commerciali percepite dai fronterizos come un'ingiusta imposizione di un governo centrale invasivo, la cui presenza nelle regioni settentrionali non assumeva altra forma, oltre all'esercito, che quella del contraresguardo e dei controlli doganali ${ }^{26}$. La merce arrivava a fiumi attraverso il confine, che sulla sponda statunitense vedeva spuntare numerosi centri urbani destinati a divenire punti nevralgici delle rotte del contrabbando verso sud. Durante la Guerra Civile Americana (1861-1865), la Confederazione si serviva del confine messicano per esportare illegalmente il cotone in Europa. Almeno fino alla fine della Guerra e alla contemporanea abolizione della schiavitù in tutti gli States, delle stesse vie del traffico di merci si avvalevano gli schiavi neri in fuga dagli Stati Uniti alla ricerca della libertà a sud del Rio Grande, dove la schiavitù era illegale.

$\mathrm{Al}$ voluminoso flusso procedente dagli Stati Uniti corrispondeva un più esiguo ma comunque significativo traffico in direzione contraria. Per decenni il commercio illegale di insetti da collezione e animali rari (specialmente di pappagalli, spesso intossicati con tequila per stordirli ed evitare così cinguettii rivelatori) ha dominato la rotta transfrontaliera verso gli States, in compagnia di altri beni di difficile reperibilità a nord del confine tra cui medicinali e prostitute ${ }^{27}$. Non solo prodotti e animali dunque, ma anche persone venivano "contrabbandate" attraverso gli stessi canali transfrontalieri, spinte ed attirate da un determinato sistema di restrizioni, divieti e tariffe. In questo sistema di traffici illeciti in rapido divenire, quelli che un secolo più tardi si sarebbero imposti all'attenzione della politica e dell'opinione pubblica di entrambe le sponde del Río Grande come i principali esempi di attraversamento illegale del confine - narcotici e migranti - già muovevano i loro primi passi transfrontalieri nella sostanziale indifferenza.

In generale, sebbene il governo messicano condannasse il contrabbando, colpevole di rovinare l'industria locale, erodere le finanze nazionali e minare l'interesse della nazione - senza considerare gli effetti morali del "mercato del vizio" che si andava consolidando - gli abitanti della frontiera lo ritenevano un'attività economica rispettabile, nonché la principale, irrinunciabile attività lavorativa praticabile. Soprattutto, il commercio illegale rappresentava un espressione del libero commercio, contrappeso positivo alle politiche commerciali restrittive imposte da Città del Messico 28.

${ }^{26}$ MORA-TORRES, Juan, op. cit., p. 35.

27 ANDREAS, Peter, op. cit., p. 31.

${ }^{28}$ MORA-TORRES, Juan, op. cit., p. 36. 
Lentamente, iniziava a farsi strada lungo le vie commerciali transfrontaliere anche la futura protagonista eccellente dell'economia illegale nordamericana: la droga. Fino agli inizi del XX secolo l'oppio e i suoi derivati, cocaina e marijuana potevano essere acquistate, vendute e consumate senza problemi negli Stati Uniti e, seppur parte del campionario di merci commerciate attraverso la frontiera, non rappresentassero una porzione significativa del commercio legale fra i due paesi. Il mercato nero dei narcotici iniziò a crearsi parallelamente al progressivo innalzamento di tariffe e barriere commerciali (specialmente sull'oppio nel 1883 e nel 1887), per trasformarsi in un'economia illecita dalle dimensioni ragguardevoli nel momento in cui le droghe iniziarono ad essere completamente bandite, cosa che avvenne negli prima con l'Opium Exclusion Act del 1909 e poi con la Harrison Narcotics Law del 1914. In modo simile a ciò che si sarebbe verificato nel decennio successivo a seguito della proibizione degli alcolici, il divieto di commercio per un bene per cui esisteva una consistente domanda creò lo spazio per un mercato nero dal valore di milioni di dollari, favorendo rapidamente l'espansione della nascente industria messicana di produzione e di esportazione di narcotici ${ }^{29}$. I commercianti erano diventati trafficanti. Sufficientemente remote ed assolate, le montagne del Sinaloa si rivelarono un ottimo territorio per la coltivazione del papavero da oppio, che divenne la base del sostentamento economico di molte famiglie di campesinos, là come in altri stati del Messico nordoccidentale (Sonora, Chihuahua, Nayarit e Durango), dove presto si concentrò il principale network di distribuzione di narcotici destinati agli Stati Uniti. Il confine si consolidò come un luogo di continua esplorazione e sperimentazione dei più ingegnosi metodi di contrabbando, in un gioco di strategia in cui il campionario di metodi creativi dispiegato nel trasporto - che giunse ad includere l'utilizzo di piccioni viaggiatori con "pacchettini" legati alle zampe, catapulte, sottomarini e tunnel, oltre a tutte le declinazioni possibili della sofisticata arte del farcire e del foderare - a più di un secolo di distanza non si è ancora esaurito ${ }^{30}$.

Nonostante il governo messicano avesse rapidamente recepita e fatta propria la politica antinarcotici del vicino settentrionale convertendola nelle leggi del 1916 (proibizione dell'importazione di oppio) e del 1923 (estensione della proibizione a tutti i narcotici), a quel tempo l'enforcement della legislazione antidroga, così come in generale l'infrastruttura doganale e la capacità di intercettare il contrabbando, era minima.

29 RECIO, Gabriela, «Drugs and Alcohol: US Prohibition and the Origins of Drug Trade in Mexico, 1910-1930 », Journal of Latin American Studies, 34, 1/2002, pp. 21-42.

$3^{\circ}$ CAMPBELL, Howard, Drug War Zone: Frontline Dispatches from the Streets of El Paso and Juárez, Austin, University of Texas Press, 2009, p. 15. 
Perciò, nonostante i divieti vigenti su entrambe le sponde del Rio Grande/Río Bravo - o per essere più precisi, proprio grazie ad essi - nella prima metà del secolo quel mercato dei narcotici tra Messico e Stati Uniti, oggi al centro delle cronache per la preoccupante violenza ad esso associata e per l’intensità con cui entrambi gli apparati statali ne perseguono l'eradicazione, era già pienamente funzionante e dinamico.

Negli anni Venti del Novecento, a seguito dell'introduzione del proibizionismo, il contrabbando di alcolici dal Messico diventò improvvisamente molto redditizio, favorendo il boom di questo settore nell'economia degli scambi commerciali tra i due paesi. Fu proprio durante il proibizionismo che all'impennata del traffico illegale di alcolici corrisposero due diversi tipi di "proliferazione organizzativa”. A sud del confine le organizzazioni criminali acquisirono la forma di cartelli con aspirazioni oligopolistiche, vere e proprie mafie che mediante l'uso estensivo di violenza e corruzione riuscirono definitivamente a prendere il controllo del mercato illecito a livello nazionale. A nord del Rio Bravo, invece, si avviò la creazione di una vera e propria infrastruttura dedicata all'interdizione delle merci di contrabbando, rappresentata in primis dalla Border Patrol, il corpo di polizia specificamente deputato all'intercettazione di tentativi illeciti di introdurre negli Stati Uniti persone e beni indesiderati, istituita nel 1924. Inizialmente i 450 agenti dislocati lungo entrambe le linee di confine statunitensi avevano come priorità l'interruzione del commercio illecito di alcolici, seguito dall'immigrazione illegale di europei e soprattutto di cinesi, cui il Chinese Exclusion Act del 1882 aveva serrato la porta d'ingresso legale. I migranti cinesi furono i primi protagonisti dei controlli migratori sul confine. Il Chinese Exclusion Act, oltre ad essere la prima legge statunitense di controllo dell'immigrazione basata sull'etnia e sulla classe sociale ${ }^{31}$, inaugurò un'epoca di progressive restrizioni migratorie, diametralmente opposta alla politica di reclutamento attivo mantenuta da Washington fino ad appena un decennio prima. Dall'altra parte del confine, Città del Messico continuava invece ad incoraggiare l'immigrazione cinese, considerata dalle autorità del Porfiriato una fondamentale leva della modernizzazione infrastrutturale del paese, anche se di qualità inferiore a quella europea ${ }^{32}$. La combinazione di questi due diversi approcci politico-giuridici fu il principale meccanismo di immigrazione cinese irregolare attraverso il confine tra Messico e Stati Uniti, allargatosi poi ad altre

${ }^{31}$ La legge ammetteva unicamente un limitato numero di visti qualificati (per studenti, insegnanti, commercianti, diplomatici), escludendo completamente i lavoratori generici. Cfr. LEE, Erika, «Enforcing the Borders: Chinese Exclusion along the U.S. Border with Canada and Mexico», The Journal of American History, 89, 1/2002, pp. 54-86.

32 Ibidem, p. 59. 
nazionalità ed assestatosi in maniera stabile dopo il 1924, quando l'Immigration Act introdusse l'obbligo del visto ponendo fine all'era della open immigration.

Nel 1906 si stimava che l'80\% dei migranti cinesi arrivati in Messico continuasse il proprio viaggio attraverso il confine settentrionale, potendo usufruire delle reti, della logistica e delle infrastrutture del contrabbando già attive nelle città fiorite su entrambe le sponde del confine anche e soprattutto grazie al dinamismo dell'economia illegale. Il prezzo dell'attraversamento del confine pagato alle "guide", oggi denominati coyotes o polleros, agenti nel sempre più richiesto di "contrabbando di persone" 33 , passò dai $25 \$$ del 1890 ai $200 \$$ del $1930^{34}$. La porta d'ingresso negli Stati Uniti rimase totalmente aperta fino alla chiusura parziale del 1917 (e parzialmente aperta fino alla legge del 1924), anno in cui si tentò di arginare il flusso di messicani in fuga da un paese scosso dalla Rivoluzione tramite l'istituzione di un test di alfabetizzazione e di una tassa di 8 dollari. Per i migranti provenienti dal Messico, un metodo rivelatosi piuttosto efficace per evadere i controlli alla frontiera, fu quello di camuffare i migranti cinesi facendoli passare per messicani o nativi americani35.

Esteban Cantú, governatore dello Stato della Baja California tra il 1915 e il 1920, fu un personaggio paradigmatico, in grado di agire nella zona grigia tra divieti e opportunità, ovvero tra la distanza fisica e politica dal centro di emanazione del potere statale e la vicinanza ad una linea di frontiera in grado di trasformare un prodotto liberamente disponibile in una rarità proibita ed agognata. Durante il suo governo, libero dai vincoli di Città del Messico, Cantú fue su propia ley: nominava autonomamente i propri funzionari, imponeva e riscuoteva le sue tasse e decideva dei dazi di importazione ${ }^{36}$. Tutto ciò, rigorosamente in dollari. Dissimulando la propria fedeltà a seconda del momentaneo prevalere di una delle diverse fazioni della Rivoluzione che stava in quel momento scuotendo il paese, il governatore costruì un impero finanziato grazie all'economia illegale transfrontaliera. I proventi del traffico di oppio, cocaina, morfina, eroina, della prostituzione e della tratta di persone, delle corse dei cavalli, delle case da gioco, cantine e tuguri di vario genere riempirono le casse dello Stato, rendendo la California messicana un vero e proprio "Eldorado del vizio", sospinto dalle proibizioni vigenti a nord dal confine e alla portata delle tasche dei

33 Il crimine di traffico di migranti, che si distingue da quello della tratta di persone per la presenza del consenso da parte del soggetto, per la durata dello sfruttamento materiale del soggetto (che normalmente termina con l'arrivo del migrante nel paese di destinazione), e per il carattere transnazionale, è sanzionato dal Protocollo alla Convenzione delle Nazioni Unite contro la criminalità organizzata transnazionale, adottata a Palermo nel 2000.

34 LEE, Erika, op. cit., pp. 59-60

35 Ibidem, p. 61

${ }^{36}$ WERNE, J. Richard, «Esteban Cantú y la soberanía mexicana en Baja California», in Historia Mexicana, XXX, 1/1980, p. 7 
visitatori statunitensi. Nell'altra direzione, armi e munizioni erano contrabbandate in Baja California, violando il decreto emanato da Wilson, che ne proibiva l'esportazione. Il caso di Cantú fu un perfetto esempio di stretto intreccio tra narcotraffico e politica, a dimostrazione di come il traffico illecito non operasse in una dimensione parallela a quella dello Stato messicano bensì di come fosse nato e cresciuto insieme ad esso.

D’altra parte, proprio grazie ai proventi di armi e oppio, Cantú fu in grado di rafforzare la sovranità sul territorio. Se è vero che il suo efficace ed insolitamente ben remunerato esercito era un baluardo di indipendenza e autonomia de facto dal centro, esso rappresentava anche un argine messicano alla temuta ingerenza statunitense in un momento di somma incertezza e instabilità nella politica nazionale, che in caso di difficoltà di fronte ad un intervento straniero non avrebbe avuto le risorse per intervenire. Oltre le speculazioni sulla reale possibilità che Washington intendesse annettere la Baja California, scenario tanto paventato dai messicani di allora quanto scartato dagli studiosi di oggi, probabilmente gli sforzi di Cantú contribuirono ad affermare la sovranità messicana sulla Baja California37. Paradossalmente, gli introiti del vizio e del contrabbando provvedevano al finanziamento dell'amministrazione californiana. I proventi dell'illecito, convertiti non solo in stipendi di funzionari pubblici ma tradotto anche in servizi, infrastrutture sociali, e più in generale utilizzati per costruire o rafforzare una presenza fisica dello Stato laddove essa mancava, assicuravano il mantenimento della pace nella regione.

Per tutto il XIX secolo e oltre, dunque, l'attraversamento del confine meridionale degli Stati Uniti non presentava particolari difficoltà. Gli sforzi delle autorità di controllo migratorio si concentravano sui punti d'entrata via mare e sul controllo degli arrivi dall'Europa e dall'Asia, lasciando che persone, beni, servizi e veicoli passassero liberamente il confine, in un'assenza quasi totale di controllo. Lungo la rete ferroviaria che collegava le località messicane di frontiera con le città economicamente più importanti degli Stati Uniti esistevano scarse possibilità di incontrare un’ispezione. Sospinta dalla volontà di controllare in particolare l'immigrazione cinese, una vera e propria infrastruttura di border enforcement fu messa in atto solo dal 1903, quando l'Immigration Service iniziò timidamente a registrare le entrate e a condurre ispezioni sui migranti presso il confine. Nel 1904 erano ottanta gli agenti deputati ad effettuare controlli migratori lungo una linea di oltre $3.000 \mathrm{~km}^{38}$. Vent'anni più tardi, con la creazione della Border Patrol, lo spazio nazionale venne riconfigurato secondo un nuovo senso di territorialità, marcato da una attenzione ai confini senza precedenti

37 Ibidem, p. 29.

${ }^{38}$ Ibidem, pp. 71, 82. 
destinata a non conoscere inversione di rotta nei decenni a venire39. Man mano che le rotte del contrabbando e degli attraversamenti illeciti si spingevano verso spazi remoti, allontanandosi dai controlli, ne trascinavano allo stesso tempo l'espansione, sospingendo l'intrusione del border enforcement in luoghi precedentemente incontrollati. Lo sviluppo dell'infrastruttura del controllo del confine all'inseguimento degli attraversamenti illeciti della frontiera incalzò l'avanzata geografica del confine sul territorio e nella vita reale delle borderlands, rendendo effettiva quella che fino a prima non era che una linea incerta segnata sulle mappe.

Lungi dall'essere in contraddizione o, come potrebbero apparire, l'uno negazione dell'altro, la pratica del contrabbando e lo Stato si sono in realtà intrecciati in molteplici modi lungo la storia della costruzione del confine tra Messico e Stati Uniti. Più che contrapporsi all'economia formale, le reti del commercio illegale appaiono ad essa inestricabilmente vincolate, esendo entrambe messe in moto dalla ferrea legge del capitalismo, che afferma che laddove vi è domanda per un bene, ci sarà qualcuno che provvederà a fornirlo, sempre che il profitto sia sufficiente a compensare i rischi. Inoltre, la stessa definizione di pratiche illegali non è esente da complessità. Allo Stato, per via della legge, spetta definire ciò che è legale e ciò che non lo è, ovvero collocare un determinato comportamento nello spazio di liceità o di illiceità. Tale azione, prendendo forma in un dato momento storico, secondo un sentire sociale e culturale localizzato nel "tempo", esprime una relazione mutevole tra lo Stato e una persona o un gruppo e ne produce lo status di illegalità, condizione che vede i propri limiti e termini di appartenenza modificarsi nel tempo. Ma tale situazione si verificava anche nello "spazio", considerando come, nel caso dei territori di frontiera tra Messico settentrionale e Stati Uniti meridionali, la presenza di un confine a dividere due giurisdizioni diverse poteva produrre (ed ha infatti prodotto) situazioni in cui lo stesso comportamento era ritenuto legittimo da una parte ma non dall'altra, come nel caso dei narcotici. Considerando poi la legge non tanto come un sistema definito di regole, ma piuttosto come un «sistema indeterminato di significati manipolato nella pratica sociale» $4^{\circ}$, le pratiche dell'economia illegale non si collocano staticamente al di fuori dello spazio di affermazione dello Stato, bensì in una zona mobile di ambiguità, di interpenetrazione e di contestazione. A questo proposito, non si può non ricordare la percezione culturale dell'illegalità prevalente nella società delle borderlands

39 NGAI, Mae, Impossible Subjects: illegal aliens in the making of modern America, Princeton, Princeton University Press, 2004.

4o McC, HEYMAN Josiah, SMART, Alan, States and Illegal Practices: an Overview, in McC HEYMAN, J. (a cura di), States and Illegal Practices, Oxford, Oxford International Publishers, 1999, p. 11. 
messicano-americane del XIX secolo, dove pratiche come il banditismo e il contrabbando erano generalmente accettate come legittime, mentre i divieti e le imposizioni esercitate da uno Stato centrale lontano apparivano come devianti ed illecite.

La storia del confine tra Messico e Stati Uniti è dunque un esempio di come l'azione dello Stato, non solo non sopprima il crimine, - ostacolata da una sovranità incompleta e da una grande distanza fisica dal centro politico -, ma lo incoraggi attivamente, creando degli spazi di opportunità, come dimostrato dalle conseguenze della proibizione del commercio di prodotti richiesti, offerti e distribuibili. Per di più, tra i gruppi che si muovono nelle e vivono grazie alle zone di illegalità esiste una relazione di mutuo parassitismo: da una parte i contrabbandieri sfruttano economicamente quel vuoto tra domanda e offerta di un bene creato dall'imposizione di una proibizione, dall'altra la burocrazia e l'apparato di enforcement la cui esistenza e proliferazione sono giustificate proprio dall'esistenza di tali gruppi.

In conclusione, non solo i network dell'illecito appaiono come intrinsecamente vincolati all'economia legale e alle strutture dello Stato, essendone parzialmente il prodotto: in alcune occasioni essi finiscono per promuovere il processo di consolidamento dello stesso. Oltre alla dinamica di produzione di istituzioni di controllo del confine - e dunque in un certo senso del confine stesso - generata dalla corsa all'inseguimento del contrabbando e degli attraversamenti illeciti, la Baja California di Esteban Cantú rappresenta un esempio di "spazio grigio" tra legalità e illegalità, di interazione complessa tra pratiche informali ed istituzioni formali, in cui le risorse generate dalla proliferazione dell'illecito sono convogliate in canali istituzionali contribuendo a sopperire alla scarsità delle finanze statali. In un panorama di debole presenza dello Stato, di proliferazione di centri di potere locali e di regimi di autonomie territoriali de facto, l'economia informale ha partecipato al rafforzamento delle istituzioni dello stato. In altre parole, «bandits helped make states, and states make bandits» ${ }^{4}$.

41 GALlaNT, Thomas W., Brigandage, Piracy, Capitalism, and State-Formation: Transnational Crime from a Historical World-System Perspective, in McC HEYMAN J. (ed.), States and Illegal Practices, Oxford, Oxford International Publishers, 1999, p. 25. 


\section{* L'autore}

Valentina Abalzati, conseguita la laurea magistrale in Scienze Internazionali e Diplomatiche nella facoltà "R. Ruffilli” di Forlì (Università di Bologna), con una tesi sulla riconfigurazione del confine tra Messico e Stati Uniti tra economie aperte e frontiere chiuse, si è trasferita a Città del Messico dove aveva precedentemente trascorso un semestre di studio presso la Universidad Nacional Autónoma de México. Attualmente lavora come analista free lance per questioni di politica e sicurezza e collabora saltuariamente con alcune riviste di politica internazionale, tra cui «Limes» e «World in Progress».

URL: < http://www.studistorici.com/progett/autori/\#Abalzati >

\section{Per citare questo articolo:}

ABALZATI, Valentina, «Contrabbandieri, banditi e guardiani. Le vie del traffico illecito nella costruzione del confine tra Messico e Stati Uniti», Diacronie. Studi di Storia Contemporanea: Contrabbandieri, pirati e frontiere: per una storia delle pratiche informali nell'America Centrale (XVII-XXI secolo), 29/04/2013,

URL:< http://www.studistorici.com/2013/04/29/abalzati_numero_13/ >

\section{Diacronie Studi di Storia Contemporanea $\beta$ www.diacronie.it}

Risorsa digitale indipendente a carattere storiografico. Uscita trimestrale. redazione.diacronie@hotmail.it

Comitato di redazione: Marco Abram - Jacopo Bassi - Luca Bufarale - Alessandro Cattunar - Elisa Grandi - Deborah Paci - Fausto Pietrancosta - Matteo Tomasoni - Luca Zuccolo

Diritti: gli articoli di Diacronie. Studi di Storia Contemporanea sono pubblicati sotto licenza Creative Commons 2.5. Possono essere riprodotti a patto di non modificarne i contenuti e di non usarli per fini commerciali. La citazione di estratti è comunque sempre autorizzata, nei limiti previsti dalla legge. 